\title{
Endoscopic transpapillary gallbladder stenting for acute cholecystitis using a novel integrated inside biliary stent and nasobiliary drainage catheter system
}

A novel integrated inside biliary stent and dedicated nasobiliary drainage catheter system (UMIDAS NB stent; Olympus Medical Systems, Tokyo, Japan) has been recently developed in Japan. The 7-Fr biliary stent placed over a 4-Fr nasobiliary drainage catheter can be deployed simultaneously using a dedicated pushing catheter over the guidewire [1] ( Fig.1). Here, we describe a case of successful endoscopic transpapillary gallbladder stenting for acute cholecystitis using this drainage system.

A 77-year-old man, who underwent endoscopic sphincterotomy for biliary stones 2 months prior, was admitted for moderate gallstone-induced cholecystitis. Emergency cholecystectomy and percutaneous gallbladder drainage were considered high risk owing to percutaneous coronary intervention 1 month earlier, followed by dual antiplatelet therapy. Therefore, we performed endoscopic naso-gallbladder drainage using the novel integrated biliary stent and nasobiliary drainage catheter system ( Fig.2). We then performed gallbladder irrigation with saline via the nasobiliary drainage catheter, as appropriate.

A cholecystogram via the nasobiliary drainage catheter performed 2 days later showed contrast medium drained from the gallbladder into the lower bile duct and duodenum (>Fig.3). After acute cholecystitis improved 7 days later, the nasobiliary drainage catheter, stretched using a 0.025-inch guidewire (VisiGlide 2; Olympus Medical Systems), was removed and the biliary stent was placed from the gallbladder to the common bile duct as a bridge to cholecystectomy under fluoroscopic guidance ( $\mathbf{F i g . 4 ,}$ - Video 1). The patient was discharged without any adverse events on post-endoscopic naso-gallbladder drainage day 10.

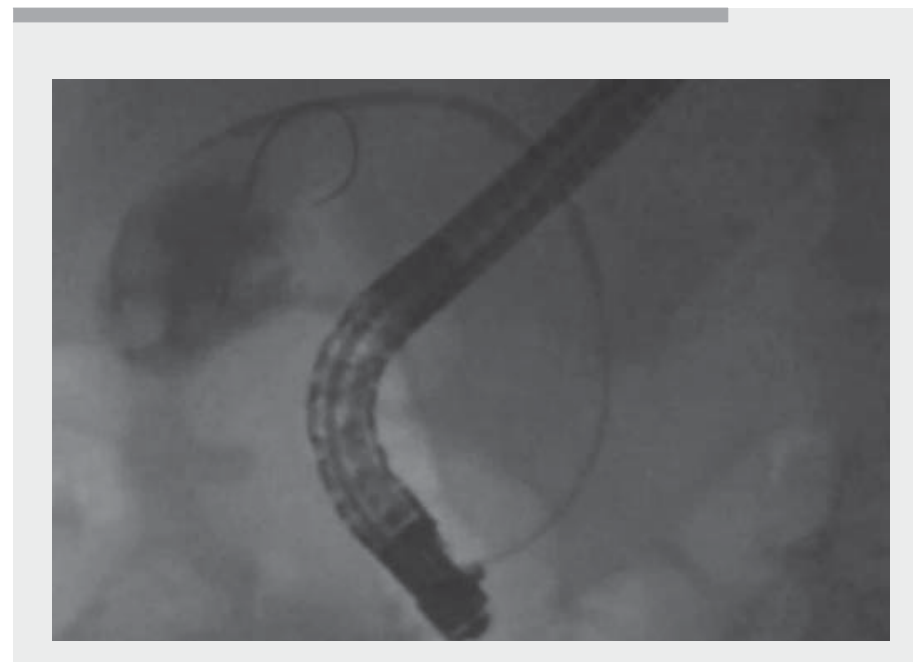

Video 1 Endoscopic transpapillary gallbladder stenting for acute cholecystitis using a novel integrated biliary stent and nasobiliary drainage catheter system.

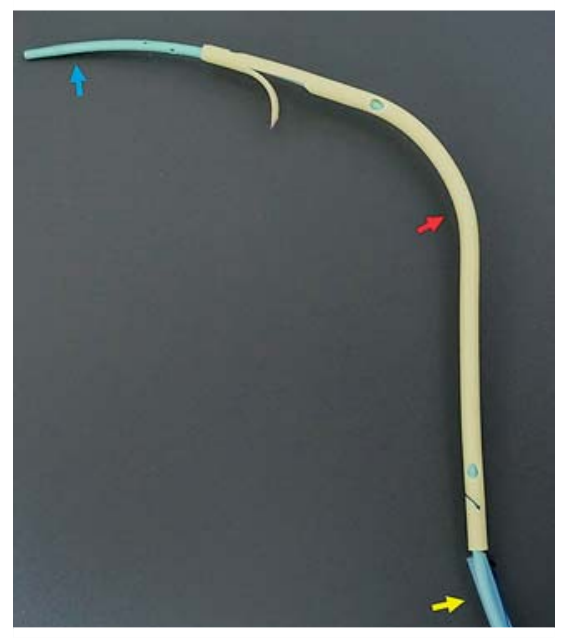

- Fig. 1 A novel integrated inside biliary stent and nasobiliary drainage catheter system that includes a 4-Fr nasobiliary drainage catheter with multiple side holes with a radiopaque marker near the tip (blue arrow), a 7-Fr inside biliary stent with a lasso (red arrow), and a 7.5- $\mathrm{Fr}$ pushing catheter with a locking system (yellow arrow).

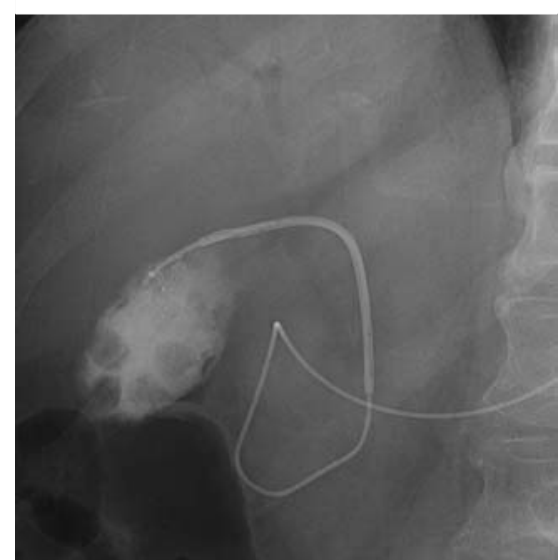

Fig. 2 Fluoroscopy shows a naso-gallbladder drainage catheter with an inside biliary stent placed.

This case suggests that this novel integrated inside biliary stent and dedicated nasobiliary drainage catheter system may be useful for endoscopic transpapillary gallbladder drainage, because it allows gallbladder irrigation via the nasobiliary drainage catheter and allows 


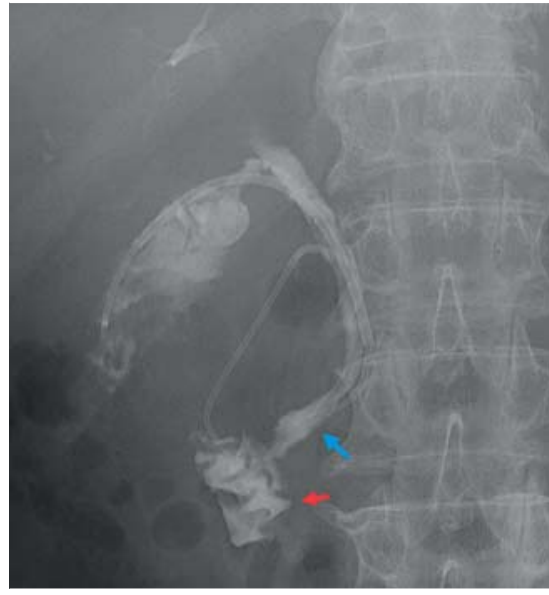

- Fig. 3 Cholecystogram via the nasogallbladder drainage catheter on postgallbladder drainage day 2 shows that the contrast medium drains from the gallbladder into the lower bile duct (blue arrow) and duodenum (red arrow).

switching from external drainage to internal stenting without another endoscopic procedure. Further studies involving many cases are needed to validate its safety and efficacy.

Endoscopy_UCTN_Code_TTT_1AO_2AH

\section{Competing interests}

The authors declare that they have no conflict of interest.

The authors

\section{Koichiro Mandai $\odot$ Takuji Kawamura, Koj} Uno, Kenjiro Yasuda

Department of Gastroenterology, Kyoto Second Red Cross Hospital, Kyoto, Japan
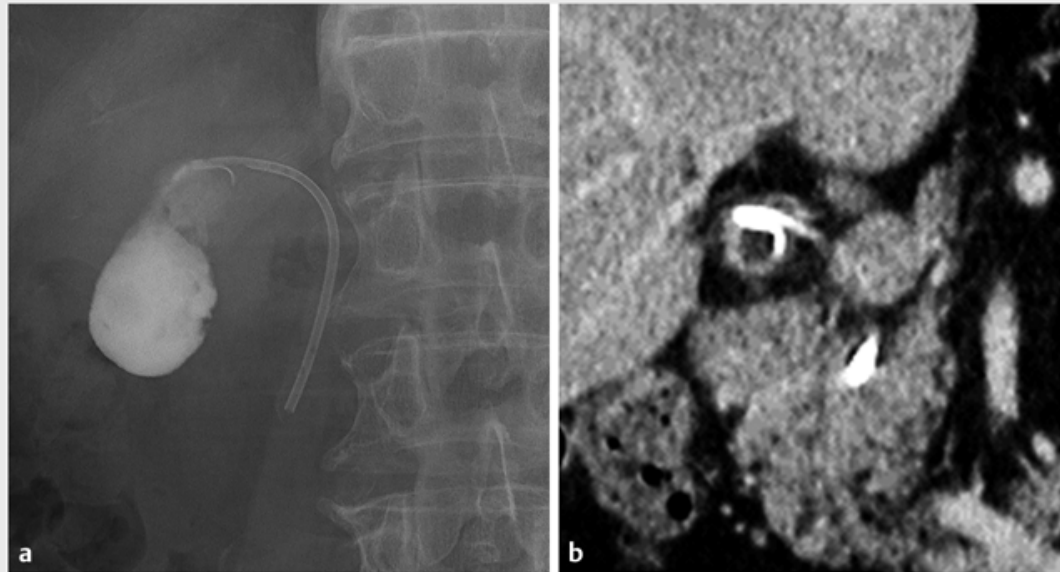

- Fig. 4 a Fluoroscopy on post-gallbladder drainage day 7 shows that the biliary stent is placed from the neck of the gallbladder to the lower bile duct. $\mathbf{b}$ A computed tomography image on post-gallbladder drainage day 9 shows that the biliary stent is placed from the neck of the gallbladder to the lower bile duct.

\section{Corresponding author}

\section{Koichiro Mandai, MD}

Department of Gastroenterology, Kyoto Second Red Cross Hospital, 355-5 Haruobicho, Kamigyo-ku, Kyoto 602-8026, Japan Fax: +81-75-256-3451 mndkchr@gmail.com

\section{Reference}

[1] Kawakami H, Itoi T. A novel integrated inside biliary stent and nasobiliary drainage catheter system for biliary drainage (with video). J Hepatobiliary Pancreat Sci 2019; 27: 149150

\section{Bibliography}

Endoscopy 2022; 54: E266-E267

DOI 10.1055/a-1512-8024

ISSN 0013-726X

published online 18.6.2021

(c) 2021. Thieme. All rights reserved. Georg Thieme Verlag KG, Rüdigerstraße 14, 70469 Stuttgart, Germany

\section{ENDOSCOPY E-VIDEOS}

https://eref.thieme.de/e-videos

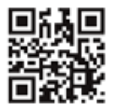

Endoscopy E-Videos is an open access online section, reporting on interesting cases and new techniques in gastroenterological endoscopy. All papers include a high quality video and all contributions are freely accessible online. Processing charges apply (currently EUR 375), discounts and wavers acc. to HINARI are available.

This section has its own submission website at

https://mc.manuscriptcentral.com/e-videos 